\title{
Evaluation of genotype resistance testing for salvage antiretroviral therapy at AIDS care centers from Ribeirão Preto, São Paulo, Brazil
}

\author{
R.J. Pires Neto ${ }^{1,2}$, J.K.B. Colares ${ }^{1}$ and B.A.L. Fonseca ${ }^{1}$ \\ ${ }^{1}$ Departamento de Clínica Médica, Faculdade de Medicina de Ribeirão Preto, Universidade de São \\ Paulo, Ribeirão Preto, SP, Brasil \\ ${ }^{2}$ Hospital São José de Doenças Infecciosas, Secretaria de Estado da Saúde, Fortaleza, CE, Brasil
}

Correspondence to: B.A.L. Fonseca, Departamento de Clínica Médica, Hospital das Clínicas, FMRPUSP, Av. Bandeirantes, 3900, 14049-900 Ribeirão Preto, SP, Brasil

Fax: +55-16-3633-0036/3633-6695. E-mail: baldfons@fmrp.usp.br

\begin{abstract}
The availability of HIV-1 genotype resistance testing (GRT) to clinicians has been insufficiently studied outside randomized clinical trials. The present study evaluated the outcome of salvage antiretroviral therapy (ART) recommended by an expert physician based on GRT in a non-clinical trial setting in Ribeirão Preto, Brazil. A prospective, open, nonrandomized study evaluating easy access to GRT at six Brazilian AIDS Clinics was carried out. This cooperative study analyzed the efficacy of treatment recommended to patients whose salvage ART was guided by GRT with that of treatment with ART based only on previous ART history. A total of 112 patients with ART failure were included in the study, and 77 of them were submitted to GRT. The median CD4 cell count and viral load for these 77 patients at baseline were (mean \pm SD) $252.1 \pm 157.4$ cells/ $\mathrm{LL}$ and 4.60 $\pm 0.5 \log _{10}$ HIV RNA copies/mL, respectively. The access time, i.e., the time elapsed between ordering the GRT and receiving the result was, on average, $71.9 \pm 37.3$ days. The study results demonstrated that access to GRT followed by expert recommendations did not improve the time to persistent treatment failure when compared to conventional salvage ART. Access to GRT in this Brazilian community health care setting did not improve the long-term virologic outcomes of HIV-infected patients experiencing treatment failure. This result is probably related to the long time required to implement ART guided by GRT.
\end{abstract}

Key words: HIV-1; Antiretroviral therapy; Salvage therapy; HIV-1 genotype resistance testing

Publication supported by FAPESP.

Received August 24, 2007. Accepted March 7, 2008

\section{Introduction}

Resistance to antiretroviral therapy (ART) is an important cause of treatment failure among HIV-1-infected patients $(1,2)$. HIV-1 genetic polymorphisms associated with drug resistance have been retrospectively shown to be associated with treatment failure, the development of AIDS, and death (3-7). Prospective studies have shown shortterm improvement in virus load suppression when genotype testing was used to guide therapy in naive and experienced patients (8-11). Other recent studies have shown either transient improvement $(12)$ or no benefit $(13,14)$ associated with the use of genotype testing, i.e., resistance assays.

Brazilian HIV-1-infected patients have had free access to ART since the early 1990's, a policy that has been associated with a decrease in HIV-related mortality and morbidity (15-17). The Brazilian Ministry of Health has recently established the National Network for HIV-1 Genotyping (RENAGENO), and has recommended HIV-1 genotype resistance testing (GRT) in association with recommendations by an expert physician to be used at AIDS 
Clinics to guide ART for patients with treatment failure (18).

Even though GRT has been validated in randomized clinical trials, there are concerns about the feasibility of its routine use and long-term efficacy in the management of HIV-1 infection in community settings. The present study investigates the impact of GRT on the selection of appropriate treatment for patients experiencing ART failure at selected Brazilian AIDS Clinics.

\section{Patients and Methods}

HIV-1-infected patients were followed at AIDS Clinics of the public health system of Ribeirão Preto, SP, Brazil. They were eligible for this study if they were older than 18 years, had been receiving a failing ART regimen containing 3 or more drugs for at least 8 weeks before enrollment, and were willing to attend regular medical appointments. A failing ART regimen was defined as a viral load (VL) of more than $3.0 \log _{10}$ HIV-1 RNA copies/mL concomitant with one or more of the following conditions: less than a 1.0 $\log _{10}$ reduction in VL 4 weeks after starting a therapy regimen, failure to suppress VL to less than 400 HIV-1

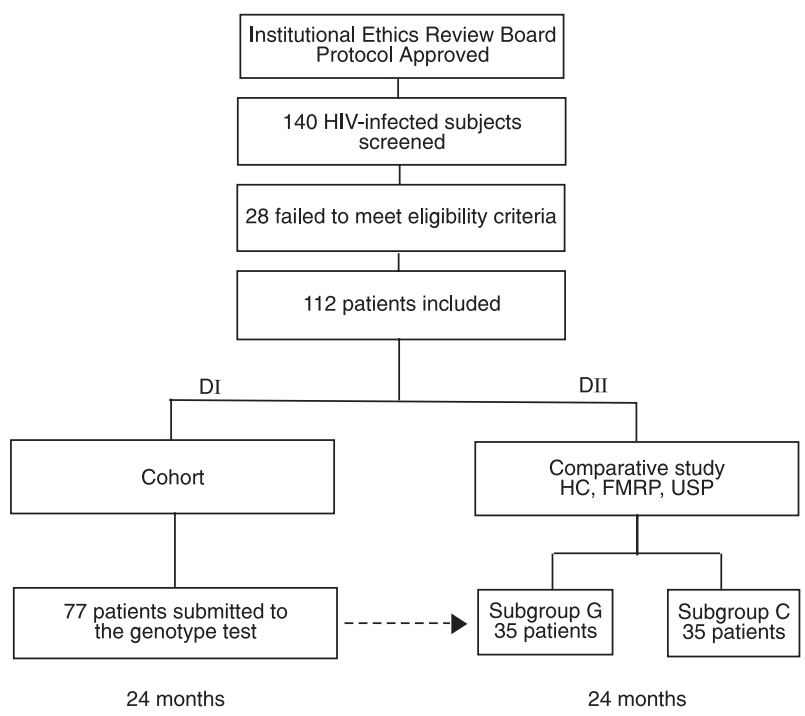

Figure 1. Flow chart of the study involving the following groups: a cohort of patients on treatment failure submitted to genotype resistance testing (GRT) to help guide a new antiretroviral therapy (ART) (DI), and a cohort of patients devised to compare the long-term efficacy of new ART guided by GRT versus new ART based on clinical judgment alone (DII). Subgroup G included patients submitted to salvage ART guided by GRT, and were screened from the DI group. Subgroup $C$ included patients submitted to salvage ART guided by clinical management without resistance testing. The DII group included patients only from the University Hospital of the School of Medicine of Ribeirão Preto, University of São Paulo (HC, FMRP, USP).
RNA copies/mL 6 weeks after starting therapy, detection of a plasma VL of more than $3.0 \log _{10}$ HIV-1 RNA copies/mL after initial suppression to less than 400 copies $/ \mathrm{mL}$, or an increase of more than $0.5 \log _{10}$ HIV-1 RNA copies $/ \mathrm{mL}$ from the nadir VL that could not be directly attributed to vaccination or intercurrent illness. The lower limit of $3.0 \log _{10} \mathrm{HIV}$ 1 RNA copies $/ \mathrm{mL}$ was chosen to minimize the effects of $\mathrm{VL}$ "blips" on the study and to reflect the performance limits of the resistance assay. Failure criteria were not mutually exclusive. All treatment failures were confirmed with a second VL measurement.

Six AIDS Clinics participated in the study, one located at the University Hospital of the School of Medicine of Ribeirão Preto, University of São Paulo (HC, FMRP, USP), and 5 being municipal health care centers. The protocol and consent form were approved by the institutional Ethics Review Board of HC, FMRP, USP. Patients were screened from December 2001 through November 2002, and those meeting the eligibility criteria were divided into two groups for analysis (Figure 1). The first group (DI) consisted of a cohort of patients undergoing ART failure who were submitted to salvage ART guided by GRT with interpretation and recommendations offered by an infectious disease physician. This study group consisted of a 24-month prospective, noncomparative study. The second group (DII) consisted of patients whose salvage ART was guided by GRT (subgroup $\mathrm{G}$ ) and by those whose salvage ART was guided by clinical management without resistance testing (subgroup $\mathrm{C}$ ). This study group consisted of a 24-month prospective, open, and comparative study designed to evaluate the long-term efficacy of salvage ART prescribed to patients undergoing ART failure. Patients in subgroup $G$ were screened from the $D I$ group. The DIl group included patients only from the $\mathrm{HC}$, FMRP, USP. The overall retention rate in both study groups was defined as the number of patients who did not reach the end-point.

Access to GRT was evaluated as the time elapsed from ordering the test by the attending physicians to receiving the final result of the test. Efficacy of GRT was evaluated by comparing salvage ART guided by GRT with conventional salvage ART based only on the previous treatment history. Participants were evaluated by routine medical appointments every 3-4 months. At each visit, plasma VL was measured using either the Quantiplex b-DNA (Chiron, Emervyille, CA, USA) or NASBA/Nuclisens (Organon Teknika, Boxtel, The Netherlands) assay according to manufacturer directions. A VL value below the detection limit of $400 \mathrm{HIV}-1$ RNA copies/mL was considered to be a response to therapy.

The study algorithm for evaluating the efficacy of GRT defined the end-point as refractory treatment failure. To 
reach an end-point, confirmed treatment failure had to be determined during two consecutive routine appointments, and a subsequent reevaluation appointment. GRT was performed with the ViroSeq HIV-1 Genotyping System kit (Applied Biosystems Inc., Foster City, CA, USA) according to manufacturer instructions.

The analysis of efficacy was performed according to the intent-to-treat principle. Baseline variables were compared using the Student $t$-test and the Fisher exact test for continuous and categorical variables, respectively. The log-rank test was used to compare the time to end-point between study subgroups. Nonparametric product-limit estimation (Kaplan-Meier) was used to estimate the time to end-point survival function. Univariate analysis was used to evaluate the effects of sex, CD4 cell count, exposure to non-nucleoside reverse-transcriptase inhibitors (NNRTIs), initial plasma HIV RNA load, and previous ART experience on the durability of virus suppression in the cohort of patients submitted to GRT. All data were collected with standardized questionnaires and entered into a database. Statistical analyses were performed using the GraphPad InStat and Prism software version 3.0 (GraphPad Software, Inc., San Diego, CA, USA).

\section{Results}

Seventy-seven adults (45.5\% women) were submitted to GRT. The median CD4 cell count at baseline \pm SD was $252.1 \pm 157.4$ cells $/ \mu \mathrm{L}$. The median VL at baseline $\pm \mathrm{SD}$ was $4.60 \pm 0.5 \log _{10}$ HIV RNA copies $/ \mathrm{mL}$. The median time of previous antiretroviral exposure \pm SD was $47.9 \pm 22.6$ months for nucleoside reverse transcriptase inhibitors, $36.5 \pm 16.5$ months for protease inhibitors, and 15.6 \pm 10.0 months for NNRTI. All classes of antiretroviral drugs available in Brazil at the time of the study had been used at least once by the patients (Table 1).

The mean turn-around time (access time) for receiving the results of the resistance test assay was $71.9 \pm 37.3$ days. The mean time elapsed from ordering GRT by clinicians from health care facilities to the date of ART change guided by the test was $132.2 \pm 70.3$ days. Only 1 patient (1.2\%) presented a wild-type virus sequence. Seven of the 77 patients $(9.1 \%)$ were not submitted to salvage ART despite availability of GRT results because 2 of them achieved undetectable HIV-1 RNA levels on their current regimen, 2 died, and 3 discontinued follow-up before salvage ART. Four other patients (5.2\%) had the ART changed before receiving the test result. Thus, salvage ART guided by GRT was initiated for 66 patients. Expert recommendations for salvage ART were adopted by attending physicians for 56 patients $(84.8 \%)$. A total of 4 patients $(6 \%)$ discontinued participation in the study for reasons other than having reached the study end-point. The estimated time to reach the end-point in the intent-to-treat analysis of these 66 patients is shown on Figure 2. Median and mean times to reach the end-point for the 62 patients kept on treatment were 316.5 and 397 days, respectively. The overall retention rate for the cohort at 12 and 24 months was $28(42.4 \%)$ and 23 patients (34.8\%), respectively.

Univariate analysis demonstrated that male gender, naive status for NNRTI, a history of less than four ART, plasma

Table 1. Antiretroviral drugs previously administered to 77 patients who had access to genotype resistance testing.

\begin{tabular}{lcr}
\hline Antiretroviral drug & Patients (N) & $\%$ \\
\hline NRTI & 77 & $100.0 \%$ \\
AZT (zidovudine) & 76 & $98.7 \%$ \\
3TC (lamivudine) & 71 & $92.2 \%$ \\
ddl (didanosine) & 54 & $70.1 \%$ \\
d4T (stavudine) & 41 & $53.2 \%$ \\
ddC (zalcitabine) & 16 & $20.7 \%$ \\
NNRTI & 28 & $36.3 \%$ \\
NVP (nevirapine) & 12 & $15.5 \%$ \\
EFV (efavirenz) & 20 & $26.0 \%$ \\
PI & 76 & $98.7 \%$ \\
SQV (sequinavir) & 22 & $28.5 \%$ \\
RTV (ritonavir) & 25 & $32.4 \%$ \\
IDV (indinavir) & 57 & $74.0 \%$ \\
NFV (nelfinavir) & 41 & $53.2 \%$ \\
APV (amprenavir) & 1 & $1.3 \%$ \\
\hline
\end{tabular}

$\mathrm{NRTI}=$ nucleoside reverse-transcriptase inhibitor; NNRTI = nonnucleoside reverse-transcriptase inhibitor; $\mathrm{PI}=$ protease inhibitor.

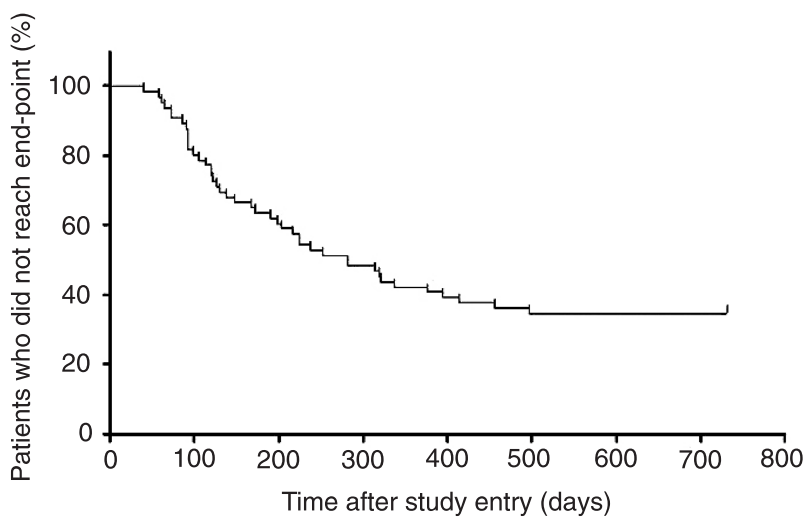

Figure 2. Estimated time to reach the end-point curve in the intent-to-treat analysis of 66 patients submitted to salvage antiretroviral therapy guided by genotype resistance testing (DI group). The estimated percentage of subjects not reaching the end-point is indicated on the $y$-axis, and the time to end-point is indicated on the x-axis. 
Table 2. Baseline demographic and clinical characteristics of 70 patients who had access to salvage antiretroviral therapy (subgroup G) or not (subgroup C) according to expert recommendations based on genotype resistance testing (GRT).

\begin{tabular}{|c|c|c|}
\hline Characteristic & Subgroup $G(N=35)$ & Subgroup $C(N=35)$ \\
\hline Age (years) & $39.6 \pm 8.7$ & $35 \pm 4.8^{*}$ \\
\hline \multicolumn{3}{|l|}{ Gender } \\
\hline Male & $20(57 \%)$ & $20(57 \%)$ \\
\hline Female & $15(43 \%)$ & $15(43 \%)$ \\
\hline \multicolumn{3}{|l|}{ Race } \\
\hline White & $23(66 \%)$ & $26(74 \%)$ \\
\hline Black & $11(32 \%)$ & $9(26 \%)$ \\
\hline Asian & $1(2 \%)$ & $0(0 \%)$ \\
\hline CD4 cell count (cells/mL)a & $209.9 \pm 185.6$ & $172.6 \pm 143.8$ \\
\hline Virus load $\left(\log _{10} \text { copies } / \mathrm{mL}\right)^{a}$ & $4.7 \pm 0.6$ & $4.8 \pm 0.8$ \\
\hline \multicolumn{3}{|l|}{ CDC HIV stage } \\
\hline A & $2(5.7 \%)$ & $1(2.8 \%)$ \\
\hline $\mathrm{B}$ & $5(14.3 \%)$ & $5(14.3 \%)$ \\
\hline C & $28(80 \%)$ & $29(82.9 \%)$ \\
\hline ART experience (months) & $50.5 \pm 22.8$ & $40 \pm 22^{*}$ \\
\hline ART experience to PI (months) ${ }^{b}$ & $39.7 \pm 15.8$ & $34.2 \pm 18.1$ \\
\hline ART experience to NRTI (months) $\mathrm{C}$ & $8.1 \pm 11.3$ & $3.9 \pm 8.1$ \\
\hline NNRTI experienced & $14(40 \%)$ & $12(34 \%)$ \\
\hline
\end{tabular}

Data are reported as mean \pm SD and as number with percent in parentheses of patients at enrollment. Categorical variables were compared using the Fisher exact test, and continuous variable was compared using the Student $t$-test $\left({ }^{*} \mathrm{P}<0.05\right.$, ${ }^{* *} \mathrm{P}$ $<0.054)$. $C D C=$ Centers for Disease Control and Prevention; ART = antiretroviral therapy; PI = protease inhibitor; NRTI = nucleoside reverse-transcriptase inhibitor; $\mathrm{NNRTI}=$ non-nucleoside reverse-transcriptase inhibitor. avalues immediately before GRT. ${ }^{\mathrm{b}} \mathrm{ART}$ experience to $\mathrm{PI}=$ period of time of previous exposure to PI. ${ }^{\mathrm{C}} \mathrm{ART}$ experience to $\mathrm{NRTI}$ = period of time of previous exposure to NRTI.

Table 3. Antiretroviral drugs previously administered to 70 patients who had access to salvage antiretroviral therapy (subgroup G) or not (subgroup C) according to expert recommendations based on genotype resistance testing.

\begin{tabular}{lcc}
\hline Antiretroviral drug & Subgroup G $(\mathrm{N}=35)$ & Subgroup C $(\mathrm{N}=35)$ \\
\hline NRTI & & \\
AZT (zidovudine) & $34(97 \%)$ & $28(100 \%)$ \\
3TC (lamivudine) & $32(91 \%)$ & $24(68 \%)$ \\
ddl (didanosine) & $26(74 \%)$ & $14(40 \%)$ \\
d4T (stavudine) & $22(62 \%)$ & $6(17 \%)$ \\
ddC (zalcitabine) & $8(22 \%)$ & \\
NNRTI & & $7(20 \%)$ \\
NVP (nevirapine) & $4(11 \%)$ & $7(20 \%)$ \\
EFV (efavirenz) & $13(37 \%)$ & $10(28 \%)$ \\
PI & & $10(28 \%)$ \\
SQV (sequinavir) & $12(34 \%)$ & $22(62 \%)$ \\
RTV (ritonavir) & $14(40 \%)$ & $8(22 \%)$ \\
IDV (indinavir) & $28(80 \%)$ & $0(0 \%)$ \\
NFV (nelfinavir) & $14(40 \%)$ & \\
APV (amprenavir) & $0(0 \%)$ & \\
\hline
\end{tabular}

Data are reported as number with percent in parentheses of patients at enrollment. $\mathrm{NRTI}=$ nucleoside reverse-transcriptase inhibitor; NNRTI = non-nucleoside reverse-transcriptase inhibitor; $\mathrm{PI}=$ protease inhibitor.
HIV-1 VL $<50,000$ RNA copies/mL, and CD4 cell count $\geq 200 / \mathrm{mL}$ were associated with longer times to reach the end-point of therapy failure when salvage ART was guided by HIV-1 GRT (Student $t$-test; $\mathrm{P}<0.05$ ).

\section{Comparative study}

A total of 70 patients were included in the comparative study, 35 in each subgroup ( $\mathrm{G}$ and $\mathrm{C}$ ). Baseline demographic data, HIV disease characteristics, and treatment histories of patients from both subgroups are summarized in Table 2. There were no significant differences in either subgroup for any of the baseline characteristics except for age, which was slightly higher in subgroup $G$. The mean times of previous ART exposure were $50.5 \pm 22.8$ and $40 \pm$ 22 months for subgroups $\mathrm{G}$ and $\mathrm{C}$, respectively. The ART previously used by both subgroups are presented in Table 3.

The overall retention rate for the cohort at 24 months was 10 (28.5\%) and 9 patients $(25.7 \%)$ for subgroups $\mathrm{G}$ and $\mathrm{C}$, respectively. A total of 6 patients, 4 from subgroup $G$ and 2 from subgroup $C$, discontinued participation in the comparative study for reasons other than having reached the study end-point. Median and mean times taken to reach the end-point were 190 and 312.5 days for subgroup $G$, and 175 and 319.8 days for subgroup $C$, respectively. $A$ comparison of survival curves using the log-rank test demonstrated no significant differences in mean time to reach the endpoint between groups (Figure 3).

\section{Discussion}

The purpose of this study was to evaluate the routine use of HIV-1 GRT in a realistic clinical practice setting through the use of GRT based on expert recommendations by an infectious disease specialist in a group of patients with ART failure attended at AIDS Clinics from Ribeirão Preto, São Paulo. Due to the characteristics of AIDS care in Brazil, the impact on ART change according to expert recommendations based on GRT results seemed to be a necessary step to be evaluated in order to 
further improve this program.

The successful use of GRT by clinicians in this study was partially compromised by the delay in the access to the test results. The mean turn-around time for receiving the results of the resistance test assay in the present study was too long. Turn-around time for the resistance test assay is a parameter never evaluated in clinical trials, except in the study of Wegner et al. (19) in which the mean time for the genotype resistance assays was 5 working days. Time from ordering resistance testing to ART change should be as short as possible. A delay may represent a risk of disease worsening since patients with treatment failure either go without treatment or remain on failing ART until the resistance test result is available. Clinical deterioration may occur and the mutation profile may change, causing the result of the resistance test to be useless. This fact was observed in our study since only approximately $35 \%$ of the patients did not reach the end-point of the study, i.e., they were not on a failing ART.

The long-term efficacy of salvage therapy guided by GRT was analyzed here in a comparative study involving 70 patients in a realistic clinical setting. No benefit regarding the clinical parameters was observed in those patients submitted to ART change based on the routine access to HIV-1 genotype testing associated with expert recommendations when compared to those patients whose ART had been changed based on their previous history of ART use. Several previously published prospective studies have shown a benefit associated with resistance testing (8-10,20). In a prospective controlled trial, $153 \mathrm{HIV}$-1-infected patients failing on combination drug regimens were randomized to receive salvage ART guided by GRT with expert advice (78 patients) versus salvage ART guided by clinical judgment (75 patients). The percentage of patients with HIV-1 RNA values $<500$ copies $/ \mathrm{mL}$ at 4,8 , and 12 weeks was 45 vs $23 \%$ $(P=0.004), 55$ vs 25\% ( $P=0.0001)$, and 34 vs $22 \%(P=$ $0.10)$, for the GRT versus no-GRT groups, respectively (8). Similar results were obtained in another prospective study where 326 HIV-1-infected patients on stable ART and with treatment failure were randomized to receive salvage ART guided by GRT (161 patients) versus salvage ART guided by clinical judgment (165 patients). The proportion of patients with plasma HIV-1 RNA < 400 copies/mL at 24 weeks was lower for those guided by GRT than for those guided by clinical judgment (48.5 and $36.2 \%$, respectively; $P<0.05$ ) (10). In addition, these two studies have suggested that the benefit of resistance testing is increased when expert interpretation of the resistance test results is used to guide therapy changes $(8,10)$.

However, another study which included a cohort of 541 HIV-1-infected patients failed to show a relatively short- term virologic improvement with the use of either phenotypic or genotypic resistance tests in combination with expert advice. Plasma HIV-1 RNA was <200 copies/mL at week 12 in 35\% of patients in the phenotyping group, $44 \%$ in the genotyping group, and $36 \%$ in the standard-of-care group (phenotyping versus standard-of-care, $P=0.918$; genotyping versus standard-of-care, $P=0.120$ ). In a subset analysis of 179 patients experiencing a first protease inhibitor failure, the percentage of patients achieving HIV1 RNA <200 copies/mL was significantly higher in the genotyping group (65\%) than in the phenotyping (45\%) and the standard-of-care groups (45\%) (genotyping versus standard-of-care, $P=0.022$ ) (13). Another study involving 450 participants failed to show that an overall improvement in the time to refractory treatment failure was associated with routine access to either genotype or phenotype resistance testing when used in a cohort of patients with a large number of enrollees with limited previous ART exposure (19).

Most of the previous studies have assessed short-term changes in viral burden, in contrast to the long-term efficacy end-point measured in the present study. This study also differs from previous ones in that the impact of resistance testing was addressed in a community health care setting, not in highly structured clinical trial settings. Because of the unique design of our study, direct outcome comparisons with other studies are difficult, but extrapolation to other community health care centers may be relevant.

Access to GRT along with expert recommendations in

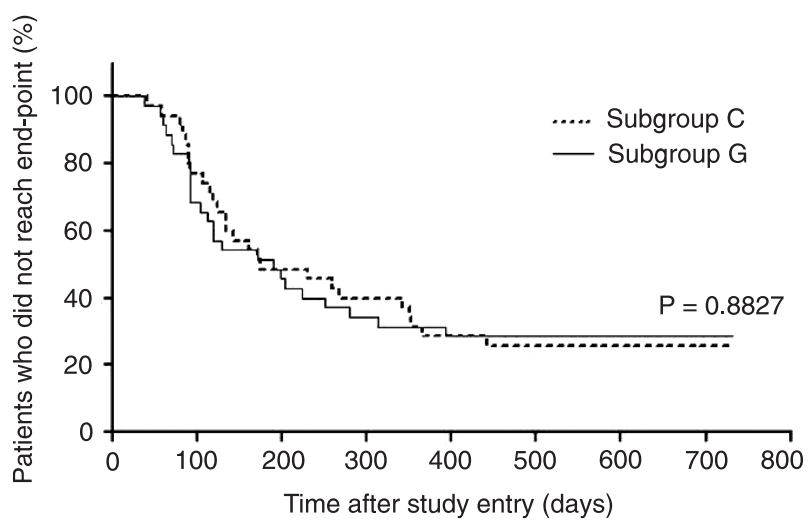

Figure 3. Estimated time to end-point curves in the intent-to-treat analysis involving 70 patients in the following subgroups: patients submitted to a new antiretroviral therapy guided by genotype resistance testing (subgroup G; 35 patients) and patients submitted to a new antiretroviral therapy guided by clinical management without resistance testing (subgroup C; 35 patients). The estimated percentage of subjects not reaching the end-point is indicated on the $y$-axis, and the time to end-point is indicated on the $x$-axis. A survival comparison using the log-rank test resulted in a $P$ value $>0.05$. 
this study was compromised in part by the delay in receiving the test results, and may have contributed to the lack of improvement of the long-term virologic outcomes in HIVinfected patients with treatment failure. At this point, it is impossible to predict that a better turn-around time would improve the results but common sense points in this direction. Measures to improve the time to return the GRT assay results to the clinicians are under study. Additional analysis of the long-term impact of GRT for salvage ART in

\section{References}

1. Hirsch MS, Conway B, D'Aquila RT, Johnson VA, BrunVezinet $F$, Clotet $B$, et al. Antiretroviral drug resistance testing in adults with HIV infection: implications for clinical management. International AIDS Society - USA Panel. JAMA 1998; 279: 1984-1991.

2. Hirsch MS, Brun-Vezinet F, Clotet B, Conway B, Kuritzkes $\mathrm{DR}$, D'Aquila RT, et al. Antiretroviral drug resistance testing in adults infected with human immunodeficiency virus type 1: 2003 recommendations of an International AIDS SocietyUSA Panel. Clin Infect Dis 2003; 37: 113-128.

3. D'Aquila RT, Johnson VA, Welles SL, Japour AJ, Kuritzkes DR, DeGruttola V, et al. Zidovudine resistance and HIV-1 disease progression during antiretroviral therapy. AIDS Clinical Trials Group Protocol 116B/117 Team and the Virology Committee Resistance Working Group. Ann Intern Med 1995; 122: 401-408.

4. Japour AJ, Welles S, D'Aquila RT, Johnson VA, Richman $D D$, Coombs RW, et al. Prevalence and clinical significance of zidovudine resistance mutations in human immunodeficiency virus isolated from patients after long-term zidovudine treatment. AIDS Clinical Trials Group 116B/117 Study Team and the Virology Committee Resistance Working Group. J Infect Dis 1995; 171: 1172-1179.

5. Miller V, Phillips A, Rottmann C, Staszewski S, Pauwels R, Hertogs $\mathrm{K}$, et al. Dual resistance to zidovudine and lamivudine in patients treated with zidovudine-lamivudine combination therapy: association with therapy failure. $J$ Infect Dis 1998; 177: 1521-1532.

6. Zolopa AR, Shafer RW, Warford A, Montoya JG, Hsu P, Katzenstein $D$, et al. HIV-1 genotypic resistance patterns predict response to saquinavir-ritonavir therapy in patients in whom previous protease inhibitor therapy had failed. Ann Intern Med 1999; 131: 813-821.

7. Lorenzi P, Opravil M, Hirschel B, Chave JP, Furrer HJ, Sax $\mathrm{H}$, et al. Impact of drug resistance mutations on virologic response to salvage therapy. Swiss HIV Cohort Study. AIDS 1999; 13: F17-F21.

8. Baxter JD, Mayers DL, Wentworth DN, Neaton JD, Hoover $\mathrm{ML}$, Winters MA, et al. A randomized study of antiretroviral management based on plasma genotypic antiretroviral resistance testing in patients failing therapy. CPCRA 046 Study Team for the Terry Beirn Community Programs for Clinical Research on AIDS. AIDS 2000; 14: F83-F93.

9. Durant J, Clevenbergh $P$, Halfon $P$, Delgiudice $P$, Porsin $S$, Simonet $P$, et al. Drug-resistance genotyping in HIV-1 therapy: the VIRADAPT randomised controlled trial. Lancet 1999; community settings is needed and should be approached by further studies on this subject, especially in countries with limited resources.

\section{Acknowledgments}

National Network of HIV Genotyping (RENAGENO); Brazilian National Program of Sexual Transmitted Diseases and AIDS, Ministry of Health, Brazil.
353: 2195-2199.

10. Tural C, Ruiz L, Holtzer C, Schapiro J, Viciana P, Gonzalez $J$, et al. Clinical utility of HIV-1 genotyping and expert advice: the Havana trial. AIDS 2002; 16: 209-218.

11. Department of Health and Human Services (DHHS). Guidelines for the use of antiretroviral agents in HIV-1-infected adults and adolescents (October 10, 2006). USA: DHHS Panel on Antiretroviral Guidelines for Adults and Adolescents; 2006.

12. Cingolani A, Antinori A, Rizzo MG, Murri R, Ammassari A, Baldini $F$, et al. Usefulness of monitoring HIV drug resistance and adherence in individuals failing highly active antiretroviral therapy: a randomized study (ARGENTA). AIDS 2002; 16: 369-379.

13. Meynard JL, Vray M, Morand-Joubert L, Race E, Descamps D, Peytavin G, et al. Phenotypic or genotypic resistance testing for choosing antiretroviral therapy after treatment failure: a randomized trial. AIDS 2002; 16: 727-736.

14. Green H, Gibb DM, Compagnucci A, Giacomet V, de Rossi $A$, Harper $L$, et al. A randomized controlled trial of genotypic HIV drug resistance testing in HIV-1-infected children: the PERA (PENTA 8) trial. Antivir Ther 2006; 11: 857-867.

15. Casseb J, Pereira Junior LC, Silva GL, Medeiros LA. Decreasing mortality and morbidity in adult AIDS patients from 1995 to 1997 in São Paulo, Brazil. AIDS Patient Care STDS 1999; 13: 213-214.

16. Marins JR, Jamal LF, Chen SY, Barros MB, Hudes ES, Barbosa AA, et al. Dramatic improvement in survival among adult Brazilian AIDS patients. AIDS 2003; 17: 1675-1682.

17. Pires Neto RJ, Lima JN, Arakaki D, Tanuri A, Arruda EAG. Síndrome da imunodeficiência adquirida. In: Lopes AC, Neto VA (Editors), Tratado de clínica médica. Vol. 3. 1st edn. São Paulo: Roca; 2006. p 4198-4239.

18. Teixeira PR, Vitoria MA, Barcarolo J. Antiretroviral treatment in resource-poor settings: the Brazilian experience. AIDS 2004; 18 (Suppl 3): S5-S7.

19. Wegner SA, Wallace MR, Aronson NE, Tasker SA, Blazes DL, Tamminga $C$, et al. Long-term efficacy of routine access to antiretroviral-resistance testing in HIV type 1-infected patients: results of the clinical efficacy of resistance testing trial. Clin Infect Dis 2004; 38: 723-730

20. Cohen CJ, Hunt S, Sension M, Farthing C, Conant M, Jacobson $\mathrm{S}$, et al. A randomized trial assessing the impact of phenotypic resistance testing on antiretroviral therapy. AIDS 2002; 16: 579-588. 